\title{
O Instituto Moinho Cultural: uma proposta de desenvolvimento sustentável cultural
}

\author{
The Instituto Moinho Cultural: a proposal for sustainable cultural development \\ Le Institute Moinho Cultural: une proposition de développement culturel \\ durable
}

\section{El Instituto Moinho Cultural: una propuesta de desarrollo sostenible cultural}

\author{
Arlinda Cantero Dorsa ${ }^{1}$ \\ Antônio Carlos Cantero Dorsa ${ }^{1}$ \\ Recebido em 04/08/2018; revisado e aprovado em 07/10/2018; aceito em 04/11/2018 \\ DOI: http://dx.doi.org/10.20435/inter.v20i2.2108
}

\begin{abstract}
Resumo: Neste trabalho, ressalta-se a importância de centralizar a sustentabilidade cultural, turística e econômica da região como premissa para o desenvolvimento local, com o objetivo de contribuir para o processo de reflexão sobre como as ações potencializadoras locais podem ser consideradas fontes de desenvolvimento sustentável de uma forma holística, ou seja: cultural, social, ecológica e econômica. Nesse contexto, este artigo focaliza o Instituto Moinho Cultural existente em Corumbá, MS, voltado ao desenvolvimento humano e econômico e ao crescimento integrado e sustentável das comunidades carentes. Conclui-se afirmando que o evento cria estratégias de interação da comunidade e firma-se como uma iniciativa exemplar de sustentabilidade, valorizando a cultura, a consciência cidadã e a solidariedade.
\end{abstract}

Palavras-chave: desenvolvimento local; sociedade sustentável; cultura.

Abstract: In this work, the importance of centralizing the cultural, tourist and economic sustainability of the region as a premise for local development is emphasized, aiming to contribute to the process of reflection on how local empowerment actions can be considered sources of development sustainable, holistic, cultural, social, ecological and economic. In this context, this article focuses on the existing Instituto Moinho Cultural in Corumbá, MS, focused on human and economic development and integrated and sustainable growth of poor communities. It concludes by stating that the event creates strategies for community interaction and stands as an exemplary sustainability initiative, valuing culture, citizen awareness and solidarity.

Keywords: localdevelopment; sustainable society; culture.

Résumé: L'accent est mis sur l'importance de la centralisation de la durabilité culturelle, touristique et économique de la région en tant que prémisse du développement local, dans le but de contribuer au processus de réflexion sur la façon dont les actions locales d'autonomisation peuvent être considérées comme des sources de développement. durable, holistique, culturel, social, écologique et économique. Dans ce contexte, cet article se concentre sur le Instituto Moinho Cultural existant à Corumbá, MS, axé sur le développement humain et économique et la croissance intégrée et durable des communautés pauvres. II conclut en déclarant que l'événement crée des stratégies d'interaction avec la communauté et se présente comme une initiative de durabilité exemplaire, valorisant la culture, la sensibilisation des citoyens et la solidarité.

Mots clés: developpement local; société durable, culture.

Resumen: En ese trabajo, se resalta la importancia de centralizar la sustentabilidad cultural, turística y económica de la región como premisa para el desarrollo local, con el objetivo de contribuir al proceso de reflexión sobre cómo las acciones potencializadoras locales pueden ser consideradas fuentes de desarrollo sostenible de una forma holística, es decir: cultural, social, ecológica y económica. En este contexto, este artículo se centra en el Instituto Molino Cultural existente en Corumbá, MS, orientado al desarrollo humano y económico y al crecimiento integrado y sostenible de las comunidades necesitadas. Se concluye afirmando que el evento crea estrategias de interacción de la comunidad y se firma como una iniciativa ejemplar de sostenibilidad, valorizando la cultura, la conciencia ciudadana y la solidaridad.

Palabras clave: desarrollo local; sociedad sostenible; cultura.

\footnotetext{
${ }^{1}$ Universidade Católica Dom Bosco (UCDB), Campo Grande, Mato Grosso do Sul, Brasil.
} 


\section{INTRODUÇÃO}

Este artigo tem por focalização o Instituto Moinho Cultural existente em Corumbá, MS, voltado ao desenvolvimento humano e econômico e ao crescimento integrado e sustentável das comunidades carentes. Nesse contexto, objetiva contribuir para o processo de reflexão sobre como as ações potencializadoras locais podem ser consideradas fontes de desenvolvimento sustentável de uma forma holística, ou seja: cultural, social, ecológica e econômica.

A investigação define-se como uma pesquisa exploratória e, em seus procedimentos técnicos, foram utilizadas pesquisas bibliográficas e documentais.

Tem-se como questão norteadora se ações públicas podem sensibilizar ações comunitárias voltadas à sustentabilidade na contemporaneidade e em resultados positivos futuros. Como referenciais teóricos, são trabalhados alguns conceitos fundamentais sobre potencialidades, desenvolvimento sustentável e sustentabilidade.

Discute então algumas temáticas importantes tais como: o desenvolvimento local, a questão da sustentabilidade, a proposta da sustentabilidade cultural e o Instituto Moinho cultural.

\section{DESENVOLVIMENTO LOCAL: OLHARES DIVERSOS}

Para a compreensão e fechamento do referencial teórico, há que se buscar a conceituação de desenvolvimento local e a sua importância para a quebra de paradigmas, visando encontrar mecanismos e procedimentos para a melhoria da qualidade de vida de uma determinada comunidade, coletividade ou grupos sociais. Para tanto, o desenvolvimento local deverá proporcionar uma transformação individual e coletiva num determinado local ou comunidade.

No desenvolvimento local, os elementos endógenos são de suma importância, pois representam a materialização dos sentimentos para o interesse coletivo e a participação dos atores sociais na definição da forma de ordenamento social em que querem viver. Em seu processo, ocorrem os elementos externos ou exógenos na figura do agente externo, que ajuda e colabora na exposição dos elementos internos, nas suas aspirações, incrementando e incentivando suas potencialidades, assim, como também, no resgate de suas referências históricas e culturais.

Albuquerque (2001) aponta os fatores que são decisivos para a ocorrência deste tipo de desenvolvimento: i) Negociação estratégica de agentes territoriais; ii) Apoio político administrativo dos gestores públicos locais; iii) Incorporação de inovações tecnológicas e inovativas no tecido empresarial e produtivo local. No entanto, para que esses fatores possam ocorrer, é necessária a formulação de políticas que busquem fomentar as potencialidades existentes no território; sendo assim, é fundamental o papel decisivo dos governos regionais e municipais como facilitadores de criação de instituições de desenvolvimento produtivo e empresarial.

É necessário então, no entendimento de Junqueira (2000), repensar primeiramente um espaço dinâmico em que a descentralização e o envolvimento comunitário possam possibilitar o surgimento de comunidades capazes de suprirem suas necessidades imediatas, ou por outro lado serem capazes de despertar as vocações locais e suas potencialidades.

As propostas de desenvolvimento local atendem a essas percepções, pois estão relacionadas às potencialidades individuais e ou coletivas que têm se fundamentado em experiências de empreendimentos, em sua maioria, pequenas, de origem local, baseadas em redes locais de cooperação, de acordo e que possuem uma "liga" formada por instituições, costumes, convenções e identidade local. 
Nesse contexto, de acordo com Fontes, Veloso e Diogo (2002), a concepção de desenvolvimento envolve o processo de expansão das liberdades reais desfrutadas pelas pessoas, e o ponto de partida para a discussão da dinâmica do desenvolvimento é o local, que pode ser uma cidade, um bairro, uma vila, um município, a beira do rio ou uma região, mas que não vise apenas ao crescimento econômico, e sim respeite o progresso social e humano.

Os autores supracitados expandem essa concepção, ao discutirem também o desenvolvimento integrado como uma forma de articulação de atores que interagem em um mesmo local, de fatores influenciadores no processo de desenvolvimento (econômicos, sociais, culturais, político-institucionais, físico-territoriais, científico-tecnológicos) além da busca de equilíbrio dinâmico nas relações que possibilitem aflorar as forças unificadoras que levem à integração e à competição.

Amplia essa discussão Vale (2007, p. 74), ao afirmar que os atores sociais:

[...] em um determinado território podem desenvolver graus variados de engajamento e comprometimento entre si e com atores externos. O grau de engajamento e comprometimento enseja o aparecimento da confiança e reciprocidade que pressupõe uma relação resultante de maior interação e dotada de uma dose de identidade compartilhada.

No processo de desenvolvimento local, o ponto de partida no município é a participação na construção de uma nova cidadania de acordo com o pensamento de Tauk Santos (1996), pois a construção das velhas identidades nacionais cede lugar à organização popular na comunidade, buscando garantir o empoderamento das populações, no sentido de torná-las protagonistas do seu desenvolvimento.

Embora não possam deixar de se basear no rumo tomado pelo desenvolvimento nacional (influência exógena), de acordo com Kleinmayer (2009), os municípios passam a ter o poder de ditar as diretrizes do seu próprio desenvolvimento, o qual deve respeitar o contexto vivenciado por cada um deles, caracterizando assim uma força endógena de desenvolvimento local.

A autonomia alcançada pelos municípios com relação ao poder da União favorece também a existência de outra descentralização. Essa, agora, dentro do próprio município, dando forma assim ao desenvolvimento local participativo, onde se torna cada vez mais essencial a participação de toda a sociedade local no estabelecimento dos parâmetros de desenvolvimento. (KLEINMAYER, 2009, p. 3).

Comunga com esse pensamento Buarque (2004, p. 25), pois, de acordo com a sua concepção, o desenvolvimento local é um processo endógeno de mudança que leva ao dinamismo econômico e à melhoria da qualidade de vida da população em pequenas unidades territoriais e agrupamentos humanos. Sendo assim, esse desenvolvimento tem, no elemento interno do setor produtivo, político e social, elementos indutores geradores capazes de promoverem uma endogeneização socioeconômica da realidade local.

Os atores econômicos, sociais e políticos têm papéis nesse processo de fundamental importância como protagonistas a partir de suas ações locais.

O Desenvolvimento local é um processo que precisa então nesse contexto, contar com o envolvimento de toda a comunidade, na elaboração e execução dos planos e projetos que tenham o lugar como objeto de intervenção. Neste sentido, a formação de uma gestão compartilhada entre os entes envolvidos na questão (Poder público local, empresas, moradores, movimentos sociais etc.) tem se constituído em um importante instrumento da governança local, ou seja, os desafios e soluções têm que ser perseguido por todos. 
(BARROS, 2009, p. 110).

O Desenvolvimento local então necessita ser visto também como um processo de gestão em que os diferentes atores, sejam eles da economia, da sociedade, da área política além de desempenharem funções importantes, precisam interagir como elementos indutores capazes de promover o empoderamento das populações.

\section{SUSTENTABILIDADE: PONTUAÇÕES CONCEITUAIS}

Além da governança, a sustentabilidade passa então a ter visibilidade, pois baseia-se em duas formas de solidariedades: a solidariedade com a geração à qual pertencemos e a solidariedade com as futuras gerações, que se apresenta como a força motriz do desenvolvimento integrado. A solidariedade do desenvolvimento deve integrar todas as formas possíveis, política, social, econômica, espacial, cultural e ambiental.

A noção de sustentabilidade vai além de problemáticas ecológicas e ambientais e pode ser vista a partir de cinco dimensões: social, econômica, ambiental, espacial e cultural.

1) Sustentabilidade social: processo de desenvolvimento baseado no ser sustentado pela maior equidade no ter, ou seja, nos direitos e nas condições das amplas massas da população, diminuindo a distância entre os padrões de vida dos mais ricos e dos mais pobres.

2) Sustentabilidade econômica: redução dos custos sociais e ambientais possibilitando uma eficiente macrossocial.

3) Sustentabilidade ambiental: aumento da capacidade de uso dos recursos naturais por meio da utilização de recursos renováveis e da limitação do uso de recursos não renováveis ou ambientalmente prejudiciais;

4) Sustentabilidade espacial: está voltada a uma configuração rural-urbana mais equilibrada;

5) Sustentabilidade cultural: respeito à continuidade das tradições culturais e até mesmo a pluralidade das soluções particulares.

Na mesma linha de pensamento, Franco (1999) não concebe a ideia de sustentabilidade relacionada às questões ecológicas e ambientais e muito menos à durabilidade, e sim à capacidade de "um processo independentemente de ser "econômico, social, cultural, político, institucional ou físico-territorial" ser sustentável quando se mantém prolongadamente no tempo" (FRANCO, 1999, p. 22).

De acordo com Rattner (1999), com relação à construção de uma sociedade sustentável, existem valores vitais como cooperação, compaixão e solidariedade que são indispensáveis para a sobrevivência e qualidade de vida humana; já com relação ao empenho humano, a participação consciente e ativa nas decisões sobre sua própria vida e a vida coletiva dá o significado necessário. Segundo o autor, porém, mesmo quando se fala em uma participação consciente e ativa, reforça-se a democracia e participação que "diretamente proporcionam direitos e oportunidades eqüitativas para acesso à informação, trabalho, serviços básicos sociais e culturais, mas não são garantias suficientes para a sustentabilidade" (RATNER,1999, p. 2).

Ao definirem sustentabilidade, Caporal e Costabeber (2004) afirmam que ela deve ser estudada objetivando um equilíbrio numa perspectiva multidimensional em sintonia com o meio ambiente e com os valores éticos e políticos. A sustentabilidade então, para que possa ser efetivada, depende de um conjunto de iniciativas entre os interlocutores e participantes sociais 
que visem a práticas sociais educativas reforçadas por sentimentos de corresponsabilidade, de valores éticos, voltadas a uma política de desenvolvimento sustentável.

A construção de uma sociedade sustentável torna-se cada vez mais um grande desafio de todas as instâncias governamentais e repousa, segundo Cruz (2002), na obtenção do desenvolvimento sustentável ancorado sobre a lógica da interface entre três importantes processos: crescimento econômico, equidade social e equilíbrio ecológico.

O mercado de trabalho seletivo e exclusivo faz com que um número considerável de pessoas procure cada vez mais empregos socialmente excluídos. Reside nessa instância, o desafio de se gerar empregos que, ao mesmo tempo, representem práticas sustentáveis e que ampliem as possibilidades da participação da população em processos decisórios fazendo crescer a responsabilidade na consciência ambiental.

A quem caberia a tarefa de fomentar políticas voltadas às práticas sociais e que visem à sustentabilidade? De acordo com Jacobi (1999, p. 32 ), cabe ao poder local a tarefa de fomentar políticas que "estimulem a participação dos indivíduos dentro de uma perspectiva intersetorial reforçando diálogos que estimulem uma compreensão dos processos a partir dos seus componentes sociais, econômicos, ecológicos, espaciais e culturais".

Experiências de poder local, bem-sucedidas, principalmente por parte de administrações municipais, mostram que a participação representa um processo continuado de democratização da vida municipal, e que segundo Jacobi (1999) visam:

i) promover iniciativas a partir de programas e campanhas especiais visando ao desenvolvimento de objetivos de interesse coletivo;

ii) reforçar o tecido associativo e ampliar a capacidade técnica e administrativa das associações;

iii) desenvolver a participação na definição de programas e projetos e na gestão dos serviços municipais.

Com relação à sustentabilidade ambiental, dentre as práticas sociais educativas, é interessante enfatizar o papel das propostas pedagógicas de educação ambiental centradas na conscientização, mudança de comportamento, desenvolvimento de competências, capacidade de avaliação e participação dos educandos sob o ponto de vista de Reigota (1998) e que são complementadas por Pádua e Tabanez (1998), que veem nelas o aumento de conhecimentos, mudança de valores e aperfeiçoamento de habilidades, condições básicas para estimular maior integração e harmonia dos indivíduos com o meio ambiente.

O desafio é, segundo Jacobi (2003), o de formular uma educação ambiental que seja crítica e inovadora, em dois níveis: formal e não formal. Assim a educação ambiental deve ser, acima de tudo, um ato político voltado para a transformação social.

\section{UMA PROPOSTA DE SUSTENTABILIDADE CULTURAL}

Com relação à sustentabilidade cultural, dentre as atividades produtivas, uma política cultural para o desenvolvimento, de acordo com Ortiz (2007, p. 27),

[...] isola determinados aspectos, privilegia alguns pontos, e atua numa direção específica. Ela pode assim propor, numa pequena comunidade, a valorização das tarefas femininas ou o incentivo de mecanismos que contribuiriam para uma melhor exploração do turismo. Um traço característico de qualquer proposta cultural é a obtenção de resultados e a possibilidade de se avaliar o que foi realizado. 
Sendo assim, a cultura exerce um papel importante para o desenvolvimento por não se restringir à dimensão econômica e ser capaz de construir ou reconstruir identidades, elevar a autoestima individual e coletiva, adicionar valor ao patrimônio existencial humano (KNOPP, 2008).

Ao se referir à questão da sustentabilidade cultural, Sachs (2008) pondera que ela se refere tanto às mudanças voltadas ao equilíbrio entre respeito à tradição e inovação, como também à capacidade de autonomia para a elaboração de um projeto nacional integrado e endógeno.

Outra contribuição sobre a questão de sustentabilidade cultural vem de Cunha (2007), ao afirmar que essa discussão precisa ser vista sob a ótica das iniciativas culturais de instituições formais e informais, destacando que se deve pensar em sistema de financiamento cultural consistente e diversificado e que agregue o poder público, a iniciativa privada e o campo da gestão cultural. Sendo assim, é necessário, segundo o autor supracitado, que alguns aspectos importantes sejam cumpridos, como:

i) ampliação da capacidade de parcerias consistentes e de longa duração;

ii) estruturação das instituições culturais fortalecidas na elaboração de projetos, planos; iii) articulação entre as várias ações implementadas com visão de médio ou longo prazo; iv) aprofundamento de campanhas nacionais visando não só à luta por recursos públicos como também pela importância da canalização de recursos privados, sendo que isto implica mudança de cultura, de valores e finalmente fortalecimento de linhas de financiamento para pesquisa e formação.

Amplia essa discussão Silva (2011, p. 6), pois para o autor:

Esses conceitos são abrangentes e remetem à tensão intensificada com a globalização entre o global e o local, dando-se destaque à importância da diversidade cultural alimentada pelos aspectos culturais locais, porém não se fechando à mescla com aspectos exteriores que podem propiciar as inovações. A sustentabilidade cultural refere-se, nesse entendimento, ao respeito que deve ser dado às diferentes culturas e às suas contribuições para a construção de modelos de desenvolvimento apropriados às especificidades de cada ecossistema, cada cultura, cada local. (SILVA, 2011, p. 6).

As políticas culturais, de acordo com Silva (2011), devem agregar, além de uma multiplicidade de agentes sociais, uma política orientada para o desenvolvimento cultural que emana da participação ativa das populações; nesse contexto, "um desafio atual é a formulação de políticas culturais que atendam à demanda de maior participação individual e local na vida cultural da sociedade" (SILVA, 2011, p. 7).

Nas propostas que emanam das políticas culturais referentes aos municípios, é fundamental perceber que a solidariedade é condição do grupo e resulta de comunhão de atitudes e de sentimentos de modo a constituir o grupo em apreço, unidade sólida, capaz de resistir às forças exteriores; Santos (2007, p. 277) reforça que ela "aperfeiçoa a vida social" e pode ser natural ou espontânea, pois decorre da própria natureza humana e voluntária, quando há o dever moral na ajuda mútua.

Entretanto, para que se alcance o desenvolvimento sustentável, é necessário haver, segundo Santos (2007), a participação dos agentes sociais em conjunto (governo, setor privado/ empresas, sociedade civil organizada e a população), para que este seja equitativo e atenda às necessidades coletivas, comuns a toda a população, de acordo com suas necessidades. 
De acordo com Giddens (1994, p. 418), o trabalho é "a realização de tarefas que envolvem esforço físico e mental, com o fim de produzir bens e serviços para a satisfação das necessidades humanas"; França Filho e Laville (2004, p. 162) complementam esse pensamento quando afirmam que "amplos setores da nossa sociedade sempre encontram seus meios de sustento material através do desenvolvimento de atividades produtivas como forma de prolongamento de práticas de solidariedade familiares e/ou comunitárias".

\subsection{Um exemplo de sustentabilidade: o Moinho Cultural, Corumbá, MS}

Sendo a cultura um dos pilares da sustentabilidade, os valores culturais individuais e grupais precisam ser levados em conta em qualquer processo. Sendo assim, a sustentabilidade é entendida como condição favorável, uma vez que permite que ações significativas para parte da população ou para um determinado grupo cultural tenham longevidade, podendo trazer efeitos mais profundos nas dinâmicas locais ou nos trabalhos de grupos e artistas. É nesse olhar que se inserem as ações promovidas pelo Instituto Moinho Cultural, na Cidade de Corumbá, MS.

Gerida pelo Instituto Homem Pantaneiro e sediado em Corumbá, MS, o Instituto Moinho Cultural, desde 2004, é simultaneamente uma escola e um espaço de artes onde a dança, música e outras artes são ferramentas valiosas para a construção e exercício da cidadania com objetivo de não só buscar talentos individuais, como também propiciar ações de combate à exclusão social no desenvolvimento de competências que promova a capacitação profissional de jovens brasileiros de Corumbá e Ladário e bolivianos de cidades fronteiriças oriundos de famílias de baixa renda.

De acordo com as informações contidas no site do Instituto Moinho Cultural ${ }^{2}$, são várias as ações realizadas para a comunidade de jovens, tais como: i) dança: do ballet clássico, à dança contemporânea e regional (Figura 1); ii) Música: da teorização ao ensino da música regional e MPB por meio de diversos instrumentos: piano, violão, viola de cocho, viola caipira, metais, percussão; canto do coral; iii) Tecnologia: informática, montagem de equipamentos, produção audiovisual; iv) atividades complementares: apoio escolar e idiomas; v) aulas de educação ambiental e patrimonial; vi) atividades socioeducativas pautadas nos quatro pilares da Unesco: aprender a aprender, aprender a ser, aprender a fazer e aprender a conviver; vii) Mostra de dança e música: realização de um espetáculo ao final do semestre de cada ano com a apresentação de danças e músicas.

Ao incluir formação profissionalizante em dança e música, o Moinho Cultural oferece também o ensino de idiomas, acompanhamento escolar além de instruções básicas de informática, educação patrimonial, educação ambiental e atividades recreativas a cerca de mais de 300 jovens.

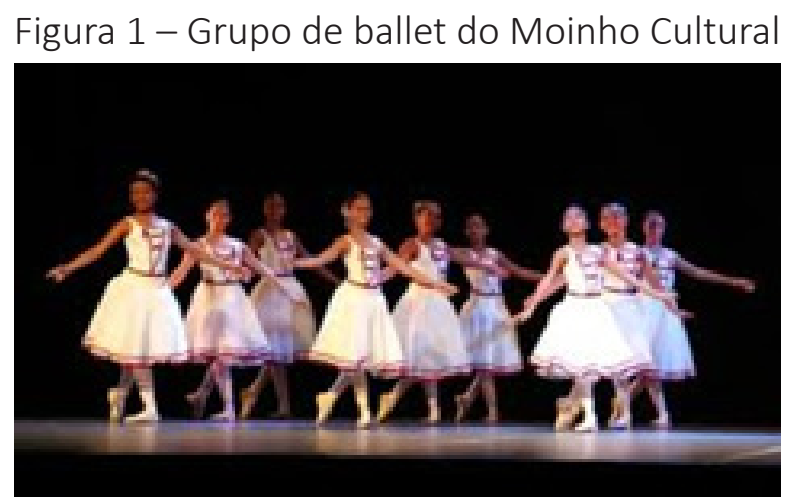

Fonte: correiodecorumba.com.br

\footnotetext{
${ }^{2}$ Site Moinho Cultural: http://www.moinhocultural.org.br/index.php?conteudo=canal\&canal_id=6
} 
Tem como exigência básica, para os alunos do projeto, a necessidade de obterem notas boas na escola e, para os pais, participarem de alguma atividade no espaço do Moinho Cultural. Nesse contexto, os jovens quando não estão na escola, encontram-se no Moinho, conforme Figura 2.

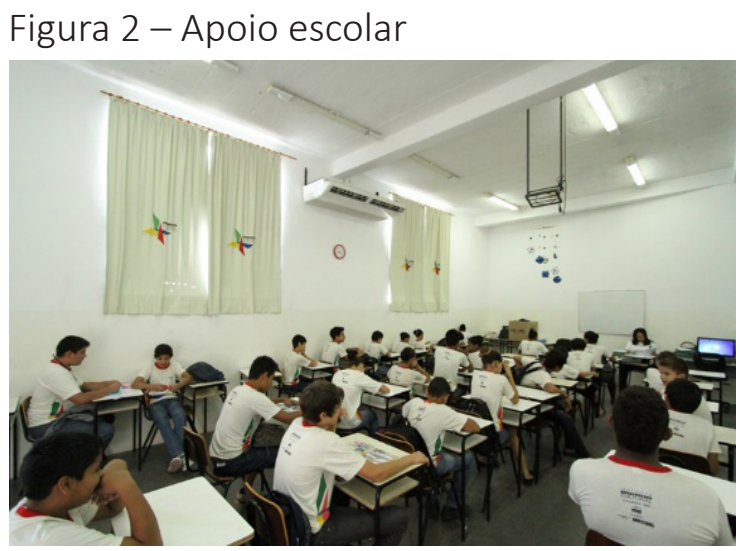

Fonte: Fundação Moinho Cultural

Conta atualmente com uma Companhia de Dança Juvenil, uma Orquestra Sinfônica Juvenil, uma Orquestra de Violões, uma Camerata, Canto Coral e um Grupo Regional de viola de cocho, instrumento típico do pantanal.

Figura 3 - Orquestra de Camerata

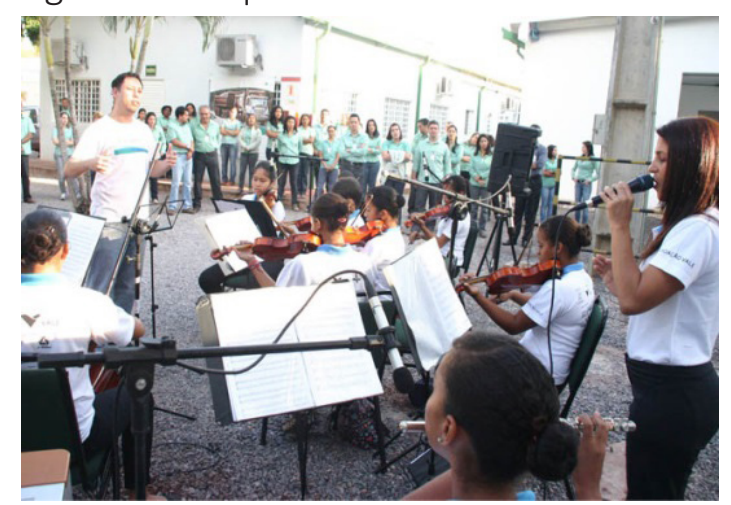

Fonte: correiodecorumba.com.br

Com relação à Mostra de dança e música, as informações contidas no site do Instituto demonstram as efetividades e visibilidades do Instituto Moinho Cultural Sul-Americano das ações culturais que têm ocorrido desde 2007, com a mobilização dos verdadeiros protagonistas: crianças e adolescentes assim como a participação de artistas brasileiros e convidados e moradores de Corumbá (MS), Ladário (MS), Puerto Suárez (Bolívia) e de Puerto Quijarro (Bolívia). Desde 2007, o Instituto tem buscado temas emblemáticos na apresentação das referidas Mostras3, tais como:

2007 - Faz de conta: Baseado nos poemas do Manoel de Barros, uma homenagem ao autor; 2008 - Quebra-cabeça: O desafio de colocar em uma única foto diferentes cabeças e culturas. Um louvor as diferenças!;

\footnotetext{
${ }^{3}$ Site Moinho Cultural: http://www.moinhocultural.org.br/index.php?conteudo=canal\&canal_id=6
} 
2009 - Rainha Nuvem: O tema foi inspirado na nuvem do mundo digital, onde tudo se pode armazenar.

2010 - Na Cabeceira dos Rios: Uma homenagem ao Pantanal, abordando o tema natureza.

2011 - Luna: As fases da lua representadas pelo astrônomo Galileu, cada uma com sua virtude e diferença.

2012 - O lado de dentro: Homenagem ao compositor e músico Geraldo Espíndola, tendo suas canções traduzidas em dança, música e arte.

2013 - O segredo da brincadeira: A proposta é reviver as brincadeiras do passado que foram esquecidas.

2014 - Moinho de artes e sonhos: Espetáculo em homenagem aos 10 anos do Moinho, com remontagem das coreografias inesquecíveis.

2015 - Sinfonia em música e dança: A Saga de uma Nota Musical.

2016 - Conto natalino e canto sul-americano.

2017 - Nossa gênese.

Assevera Albuquerque (2001) que é indispensável uma "atuação dos governos territoriais locais e regionais como catalisadores na criação do "entorno inovador", seja ele institucional, social, econômico, político e cultural, e que possa impulsionar o desenvolvimento do potencial empresarial e produtivo territorial.

É efetiva, nesse contexto, a parceria com a Fundação Vale, Oi Futuro, SESC, Itaú Cultural, Enersul, Tractebel, Andorinha, prefeitura de Corumbá e Ladário, Unesco, Ministério da Cultura e Governo Federal, para que as ações e programas sociais efetivados no Moinho Cultural possam ser voltadas ao desenvolvimento humano e econômico e para o crescimento integrado e sustentável das comunidades carentes que fazem parte do projeto.

\section{CONSIDERAÇÕES PARCIAIS}

Ao potencializar o município de Corumbá como referência cultural, com a estimulação e apoio ao crescimento interno, ao envolver a população e os municípios vizinhos, o Instituto Moinho Cultural cria estratégias de interação da comunidade e firma-se como uma iniciativa exemplar de sustentabilidade, valorizando a consciência cidadã e a solidariedade.

É relevante afirmar o valioso benefício do Moinho, ao beneficiar crianças do município de Corumbá e Ladário, como também das cidades bolivianas de Puerto Suarez e Puerto Quijarro, possibilita, assim, um intercâmbio voltado não só à formação artística e cultural como também à conquista da autonomia, à valorização pessoal e ao empoderamento de cada ator envolvido.

Para a subsistência do Instituto Moinho Cultural, têm sido fundamentais as parcerias com empresas, o apadrinhamento individual, assim como a contribuição voluntária de pessoas que se envolvem e se sensibilizam com as ações efetivadas pelo Instituto.

A participação nos projetos desenvolvidos pelo Instituto, propiciam aos participantes a efetivação dos direitos voltados à vida, à saúde, à alimentação, à arte, à dignidade, ao respeito, à convivência familiar e comunitárias. 


\section{REFERÊNCIAS}

ALBUQUERQUE, F. Desenvolvimento econômico local. Rio de Janeiro: BNDES, 2001.

BARROS, M. J. B. Mineração, finanças públicas e desenvolvimento local no município de Barcarena-Pará. Orientador: João Márcio Palheta da Silva. Dissertação (Mestrado em Geografia) - Universidade Federal do Pará, Belém, PA, 2009. Disponível em: http://repositorio.ufpa.br/jspui/bitstream/2011/5023/1/ Dissertacao_MineracaoFinancasPublicas.pdf. Acesso em: 19 abr. 2018.

BUARQUE, S. C. Construindo o desenvolvimento local sustentável. Rio de Janeiro: Garamond, 2004.

CAPORAL, F. R.; COSTABEBER, J. A. Agroecologia e Extensão Rural: contribuições para a promoção do Desenvolvimento Rural Sustentável. Brasília: MDA/SAF/DATER- IICA, 2004.

CUNHA, M. H. Gestão cultural: desafios de um novo campo profissional. Revista Observatório Itaú Cultural - OIC, São Paulo, n. 2, p. 72-79, maio/ago. 2007.

CRUZ, F. O. Reflexões sobre a sustentabilidade social, cultural e ambiental das atividades turísticas no Brasil. In: CONGRESO INTERNACIONAL DEL CLAD SOBRE LA REFORMA DEL ESTADO Y DE LA ADMINISTRACIÓN PÚBLICA, 7., Lisboa, Portugal, 8-11 out. 2002. Anais [...]. Lisboa: [s.I.], 2002.

FRANÇA FILHO, G. C.; LAVILLE, J. Economia solidária: uma abordagem internacional. Porto Alegre: UFRGS, 2004. 199 p.

FRANCO, A. Somente o desenvolvimento sustentável pode superar a pobreza no Brasil. Revista da Promoção da Saúde, Brasília, ano 1, n. 2, p. 15-8, nov./dez. 1999.

FONTES, A.; VELLOSO, M.; DIOGO, P. N. A estratégia de desenvolvimento local proposta pelo Programa Comunidade Ativa: potencialidades e entraves do DLIS. Rio de Janeiro: [s.n.], maio 2002. Disponível em: http://www.eclac.org/mujer/curso/fontes.pdf. Acesso em: 14 abr. 2017.

GIDDENS, Anthony. Sociología. Madrid: Alianza, 1994.

INSTITUTO MOINHO CULTURAL. Corumbá, [s.d.]. Disponível em: http://www.moinhocultural.org.br/index. php?conteudo=canal\&canal_id=6. Acesso em: 23 mar. 2018.

JACOBI, P. Educação ambiental, cidadania e sustentabilidade. Cadernos de Pesquisa, São Paulo, n. 118, p. 189-205, mar. 2003.

JACOBI, P. Poder Local, Políticas Sociais e Sustentabilidade. Saúde e Sociedade, São Paulo, v. 8, n. 1, p. 31 48, jan./fev. 1999. Disponível em: http://www.scielo.br/pdf/sausoc/v8n1/04.pdf. Acesso em: 15 mar. 2018.

JUNQUEIRA, L. A. P. Intersetorialidade, transetorialidade e redes sociais na saúde. Revista de Administração Pública, Rio de Janeiro, v. 34, n. 6, p. 35-45, 2000

KLEINMAYER, L. A. M. A participação dos atores locais no processo de desenvolvimento local sustentável do município de balneário Piçarras-SC. Orientador: Lafaiete Santos Neves. 2009. Dissertação (Mestrado em Organizações e Desenvolvimento) - FAE-Centro Universitário, Curitiba, PR, 2009.

KNOPP, G. C. Cultura e desenvolvimento local: um estudo do Programa Bairro-Escola na cidade de Nova Iguaçu. Orientador: Marcelo Milano Falcão Vieira. 2008. Dissertação (Mestrado em Administração)- Escola Brasileira de Administração Pública e de Empresas, Fundação Getúlio Vargas (FGV), Rio de Janeiro, RJ, 2008. Disponível em http://virtualbib.fgv.br/dspace/handle/10438/3304. Acesso em: 21 mar. 2018. 
ORTIZ, R. Cultura e desenvolvimento. In: CAMPUS EUROAMERICANO DE COOPERAÇÃO CULTURAL, 5., 2007, Almada, Portugal. Anais Eletrônicos [...]. Almada: CEC, 2007.

PÁDUA, S.; TABANEZ, M. (Org.). Educação ambiental: caminhos trilhados no Brasil. São Paulo: Ipê, 1998.

RATTNER, H. Sustentabilidade- uma visão humanista. Ambiente e Sociedade, Campinas, n. 5, jul./dez. 1999. Disponível em: http://www.scielo.br/scielo.php?script=sci_arttext\&pid=S1414-753X1999000200020\&ln g=pt\&nrm=iso. Acesso em: 24 mar. 2018.

REIGOTA, M. Desafios à educação ambiental escolar. In: JACOBI, P. et al. (Org.). Educação, meio ambiente e cidadania: reflexões e experiências. São Paulo: SMA, 1998. p. 43-50.

SANTOS, J. L. C. Planejando o desenvolvimento local sustentável. 2007. Disponível em: http://sburbanismo. vilabol.uol.com.br/artigo_desust.htm\#_ftn1. Acesso em: 10 mar. 2018.

SACHS, I. Caminhos para o desenvolvimento sustentável. 3. ed. Rio de Janeiro: Garamond, 2008.

SILVA, L. S. Sustentabilidade na cultura - da diversidade cultural à sustentação financeira. Revista Internacional Políticas Culturais, [S.I.], 2011. Disponível em: http://culturadigital.br/politicaculturalcasaderuibarbosa/ files/2011/11/Liliana-Sousa-Silva.pdf. Acesso em: 15 mar. 2018.

TAUK SANTOS, M. S. Globalização e práticas culturais: o local enquanto espaço de (re)construção da cidadania. In: COLLOQUE FRANCO BRÉSILIEN FCIC/INTERCOM, 4., 1996. Anais [...]. Grenoble: Université Stendhal, 1996.

VALE, G. M. V. Territórios vitoriosos: o papel das redes organizacionais. Rio de Janeiro: SEBRAE; Garamond Universitária, 2007.

\section{Sobre os autores:}

Arlinda Cantero Dorsa: Doutora em Língua Portuguesa. Áreas de atuação: linguagens, cultura, diversidade. E-mail: acdorsa@uol.com.br, Orcid: http://orcid.org/0000-0002-1120-0273

Antônio Carlos Cantero Dorsa: Administrador e mestrando em Desenvolvimento Local na Universidade Católica Dom Bosco (UCDB). E-mail: accdorsa@gmail.com, Orcid: http://orcid.org/0000-0002-2087-8772 
